\title{
Analysis and Design of Output Feedback Control Systems in the Presence of Actuator Saturation
}

\author{
Wei Guan and Guang-Hong Yang
}

\begin{abstract}
In this paper, a dynamic output feedback controller design approach based on cone complementary linearisation procedure is proposed for linear time-invariant (LTI) systems with actuator saturation. Firstly, the estimation of domain of attraction is given. Then, a design method to find a larger estimation of domain of attraction is presented. In the process of design, nonconvex conditions are obtained, so a cone complementary linearisation procedure is exploited to solve the nonconvex feasibility problem. Two examples are given to illustrate the efficiency of the design method.
\end{abstract}

\section{INTRODUCTION}

Control systems with actuator saturation are often encountered in practice. When actuator saturation occurs, global stability of an otherwise stable linear closed-loop system can not in general be ensured (see, [1], [2], [3]). And the problem of estimating the domain of attraction for a system with a saturated linear feedback was studied by many researchers in the last few years and various methods have appeared.

Model predictive control (MPC) is an effective control algorithm for dealing with actuator saturation. Over the last few years, many formulations were developed for the stability of MPC (see, [4], [5]). Enlargement of the domain of attraction was achieved in ([6], [7], [8], [9], [10]). Anti-windup research was largely discussed and many constructive design algorithms were formally proved to induce suitable stability properties (see, [11]-[17]). Many of these constructive approaches relied on sector condition and Sprocedure techniques and provided LMIs for the anti-windup compensator design.

In some papers, notion of invariant set and LMI-based optimization approaches were proposed to estimating the stability regions by using quadratic Lyapunov functions and the Lur'e-type Lyapunov functions. The notion of invariant set played a very important role in the study of systems with state and control constraint ([18]-[21]). In [22], the modeling of the nonlinear behavior of the system under saturation was made by using a polytopic differential inclusion and

This work was supported in part by the Funds for Creative Research Groups of China (No. 60521003), the State Key Program of National Natural Science of China (Grant No. 60534010), National 973 Program of China (Grant No. 2009CB320604), the Funds of National Science of China (Grant No. 60674021), the 111 Project (B08015) and the Funds of PhD program of MOE, China (Grant No. 20060145019).

Wei Guan is with the Department of Automation, Shenyang Institute of Aeronautical Engineering, Shenyang 110136, China; he is also with the College of Information Science and Engineering, Northeastern University, Shenyang, Liaoning, 110004, China. guanweihaha@163.com

Guang-Hong Yang is with the College of Information Science and Engineering, Northeastern University, Shenyang, Liaoning, 110004, China. yangguanghong@ise.neu.edu.cn quadratic Lyapunov functions. In [23], system

$$
\dot{x_{p}}=A_{p} x_{p}+B_{p} \sigma(u)
$$

with a given linear state feedback $u=F x_{p}$ was considered.

For determining if a given ellipsoid is contractively invariant, the authors described a condition which is based on the circle criterion or the vertex analysis, and LMI-based methods were presented for constructing state feedback laws $F$ in [23].

However, in practice, system state $x_{p}$ is not measurable in general. Our main work is to extend the state feedback results [23] to the case of dynamic output feedback. In this paper, a dynamic output feedback controller design approach based on cone complementary linearisation procedure is proposed for linear time-invariant (LTI) systems with actuator saturation. Firstly, the estimation of domain of attraction is presented. In addition, a method of controller design for LTI systems with actuator saturation is described. In the process of design, nonconvex conditions are obtained, so a cone complementary linearisation procedure is exploited to solve the nonconvex feasibility problem.

The paper is organized as follows. Problem statement is given in Section 2. A condition for set invariance is presented for LTI systems with actuator saturation in Section 3. The proposed estimation of domain of attraction is presented in Section 4. A controller design method based on cone complementary linearisation procedure is given in Section 5. And in Section 6 two examples are given to illustrate the efficiency of the design method. The paper will be concluded in Section 7.

The notations in this paper are standard. $R^{n}$ denotes $n$-dimensional real space. $R^{m \times n}$ is the set of real $m \times n$ matrices. The transpose of a real matrix $M$ is denoted by $M^{T}$. For matrix $M \in R^{n \times n}$, its trace is denoted by $\operatorname{tr}(M)$. $A>0(A \geq 0)$ means that the matrix $A$ is positive definite (semi-definite).

\section{PROBLEM STATEMENT AND PRELIMINARIES}

Consider LTI plant $\Sigma$ described by

$$
\Sigma: \quad \begin{aligned}
\dot{x_{p}} & =A_{p} x_{p}+B_{p} \sigma(u) \\
y & =C_{p} x_{p}+D_{p} \sigma(u)
\end{aligned}
$$

where $x_{p} \in R^{n_{p}}$ is the plant state, $y \in R^{n_{y}}$ is the measurement output, and $\sigma(u) \in R^{n_{u}}$ is the saturated control input. It is assumed that

(A1) the triple $\left(A_{p}, B_{p}, C_{p}\right)$ is stabilizable and detectable,

(A2) the matrices $B_{p}^{T}$ and $C_{p}$ have full row rank, 
(A3) $D_{p}=0$.

Assumption (A3) helps to simplify the derivation, and it can be satisfied by redefining the output variable.

The actuator nonlinearity with the consideration of a piecewise-linear saturation is described as

$$
\sigma\left(u_{i}\right)= \begin{cases}u_{i}, & \left|u_{i}\right| \leq u_{i}^{\max }, \\ \operatorname{sign}\left(u_{i}\right) u_{i}^{\max }, & \left|u_{i}\right|>u_{i}^{\max }\end{cases}
$$

for $i=1,2, \ldots, n_{u}$. Here we have slightly abused the notation by using $\sigma$ to denote both the scalar valued and the vector valued saturation functions.

In this paper, we will consider the dynamic output feedback controller which is given by

$$
\begin{aligned}
\Sigma_{k}: \quad \dot{x_{k}} & =A_{k} x_{k}+B_{k} y \\
u & =C_{k} x_{k}+D_{k} y
\end{aligned}
$$

where $x_{k} \in R^{n_{k}}$ is the state of system (4).

Applying the controller (4) to the system (2), the following closed-loop system $\Sigma_{d}$ can be obtained

$$
\begin{aligned}
\Sigma_{d}: \quad \dot{x} & =A_{c l} x+B_{c l} \sigma(u) \\
u & =C_{c l} x
\end{aligned}
$$

where $x=\left[\begin{array}{ll}x_{p}^{T} & x_{k}^{T}\end{array}\right]^{T}, x \in R^{n}$ with $n=n_{p}+n_{k}$, and

$A_{c l}=\left[\begin{array}{cc}A_{p} & 0 \\ B_{k} C_{p} & A_{k}\end{array}\right], B_{c l}=\left[\begin{array}{c}B_{p} \\ 0\end{array}\right], C_{c l}=\left[\begin{array}{ll}\left(D_{k} C_{p}\right) & C_{k}\end{array}\right]$.

For a matrix $C_{c l} \in R^{n_{u} \times n}$, denote the $i$ h row of $C_{c l}$ as $C_{c l i}$, define

$$
\wp\left(C_{c l}\right)=\left\{x \in R^{n}: \quad\left|C_{c l i} x\right| \leq 1, \quad i \in\left[1, n_{u}\right]\right\},
$$

then $\wp\left(C_{c l}\right)$ is the region in the state space where saturation does not occur.

For $x(0)=x_{0} \in R^{n}$, denote the state trajectory of system (5) as $\psi\left(t, x_{0}\right)$. Then the domain of attraction of the origin is

$$
\ell:=\left\{x_{0} \in R^{n}: \quad \lim _{t \rightarrow \infty} \psi\left(t, x_{0}\right)=0\right\} .
$$

Let $P \in R^{n \times n}$ be a positive-define matrix. Denote

$$
\varepsilon(P, \rho)=\left\{x \in R^{n}: \quad x^{T} P x \leq \rho\right\} .
$$

Let $V(x)=x^{T} P x$. If $\dot{V}(x)=2 x^{T} P\left(A_{c l} x+B_{c l} \sigma\left(C_{c l} x\right)\right)$ for all $x \in \varepsilon(P, \rho) \backslash\{0\}$, the ellipsoid $\varepsilon(P, \rho)$ is contractively invariant. Clearly, if $\varepsilon(P, \rho)$ is contractively invariant, then it is inside the domain of attraction.

Let $\mathbf{V}$ be a set of $n_{u} \times n_{u}$ diagonal matrices whose diagonal elements are either 1 or 0 . There are $2^{n_{u}}$ elements in $\mathbf{V}$ and we denote its elements as $V_{i}, i \in\left[1,2^{n_{u}}\right]$. Denote $V_{i}^{-}=I-V_{i}$. It is easy to see that $V_{i}^{-} \in \mathbf{V}$. The following propositions will be useful for the development of the main results of this paper.

Proposition 1 ([3]) Let $u, v \in R^{n_{u}}$ with $u=\left[u_{1}, u_{2}, \ldots, u_{n_{u}}\right]^{T}$ and $v=\left[v_{1}, v_{2}, \ldots, v_{n_{u}}\right]^{T}$. Suppose that $\left|v_{i}\right| \leq 1$ for all $i \in$ $\left[1, n_{u}\right]$.
Then,

$$
\sigma(u) \in \operatorname{co}\left\{V_{i} u+V_{i}^{-} v: i \in\left[1,2^{n_{u}}\right]\right\},
$$

where $c o$ denotes the convex hull.

A multivariable circle criterion is applied to estimate the domain of attraction for system (1), with a given state feedback $u=F x_{p}$, in the following proposition.

Proposition 2 ([23]). Given a ellipsoid $\varepsilon\left(P_{p}, \rho\right), P_{p} \in$ $R^{n_{p} \times n_{p}}$, if there exists an $D \in R^{n_{u} \times n_{p}}$, such that

$$
\begin{aligned}
\left(A_{p}+\right. & \left.B_{p}\left(V_{i} F+V_{i}^{-} D\right)\right)^{T} P_{p} \\
& +P_{p}\left(A_{p}+B_{p}\left(V_{i} F+V_{i}^{-} D\right)\right)<0
\end{aligned}
$$

for all $V_{i} \in \mathbf{V}$ and $\varepsilon\left(P_{p}, \rho\right) \subset \wp(D)$, i.e., $\left|D_{i} x_{p}\right| \leq 1$ for all $x_{p} \in$ $\varepsilon\left(P_{p}, \rho\right), i \in\left[1, n_{u}\right]$, then $\varepsilon\left(P_{p}, \rho\right)$ is a contractively invariant set.

Now, the problems under consideration in this paper are described as follows:

Problem 1 Assume that the controller (4) is given, for system (2) with actuator saturation, find an estimate of the domain of attraction.

Problem 2 For system (2) with actuator saturation, design a controller (4) such that the estimated domain of attraction is maximized respect to a prescribed bounded convex set.

\section{A CONDITION FOR SET INVARIANCE}

Assume that the standard dynamic output feedback controller has been designed. Then, for system (5) controlled by designed controller, the following theorem is presented to estimate the domain of attraction of the origin.

Theorem 1 Given a ellipsoid $\varepsilon(P, \rho), P \in R^{n \times n}$, if there exists an $H \in R^{n_{u} \times n}$, such that

$$
\begin{aligned}
\left(A_{c l}+\right. & \left.B_{c l}\left(V_{i} C_{c l}+V_{i}^{-} H\right)\right)^{T} P \\
& +P\left(A_{c l}+B_{c l}\left(V_{i} C_{c l}+V_{i}^{-} H\right)\right)<0
\end{aligned}
$$

for all $V_{i} \in \mathbf{V}$ and $\varepsilon(P, \rho) \subset \wp(H)$, i.e., $\left|H_{i} x\right| \leq 1$ for all $x \in$ $\varepsilon(P, \rho), i \in\left[1, n_{u}\right]$, then $\varepsilon(P, \rho)$ is a contractively invariant set.

Proof It is similar to the proof of Theorem 1 in [23], and omitted here.

Remark 1 Proposition 2 presents a solution to the actuator saturation problem for the state feedback case, where all states of the considered systems are assumed to be measurable. When the assumption is not valid and only system outputs are available for dynamic output feedback controller designs, Theorem 1 presents a method to solve the actuator saturation problem for the dynamic output feedback case, which is an extension of Proposition 2.

\section{ESTIMATION OF THE DOMAIN OF ATTRACTION}

From Theorem 1, we can obtain various ellipsoids satisfying the set invariance condition. So, how to choose the "largest" one of them becomes an interesting problem. In this section, we will give a method to find the "largest" ellipsoids.

The following definition will be used in the sequel.

Definition 1 ([24], [25], [26]). Define $X_{R}$ is a prescribed bounded convex set. $X_{R}=\varepsilon(R, 1)=\left\{x \in R^{n \times n}: \quad x^{T} R x \leq\right.$ $1\}, \quad R>0$ or $X_{R}=c o\left\{x_{1}, x_{2}, \ldots, x_{l}\right\}$. For a set $S \in R^{n}$, $\alpha_{R}(S)=\sup \left\{\alpha>0: \quad \alpha X_{R} \subset S\right\}$. 
In Theorem 1, a condition for the set $\varepsilon(P, \rho)$ to be inside the domain of attraction is given. With the above shape reference sets, we can choose from all the $\varepsilon(P, \rho)$ 's that satisfy the condition of Theorem 1 such that the quantity $\alpha_{R}(\varepsilon(P, \rho))$ is maximized. The problem can be formulated as follows

$$
\begin{aligned}
& \sup _{P>0, \rho, H} \alpha \\
& \text { s.t. } \quad(a) \quad \alpha X_{R} \subset \varepsilon(P, \rho) \text {, } \\
& \text { (b) }\left(A_{c l}+B_{c l}\left(V_{i} C_{c l}+V_{i}^{-} H\right)\right)^{T} P \\
& +P\left(A_{c l}+B_{c l}\left(V_{i} C_{c l}+V_{i}^{-} H\right)\right)<0 \text { for all } V_{i} \in \mathbf{V}, \\
& \text { (c) } \varepsilon(P, \rho) \subset \wp(H) \text {. }
\end{aligned}
$$

If the given shape reference set $X_{R}$ is a polyhedron as defined in Definition 1, then Constraint (a) is equivalent to

$$
\alpha^{2} x_{e}^{T}\left(\frac{P}{\rho}\right) x_{e} \leq 1 \Leftrightarrow\left[\begin{array}{cc}
1 / \alpha^{2} & x_{e}^{T} \\
x_{e} & \left(\frac{P}{\rho}\right)^{-1}
\end{array}\right] \geq 0,
$$

for all $e \in[1, l]$. If $X_{R}$ is a ellipsoid $\varepsilon(R, 1)$, then (a) is equivalent to

$$
\frac{R}{\alpha^{2}} \geq \frac{P}{\rho} \Leftrightarrow\left[\begin{array}{cc}
\left(1 / \alpha^{2}\right) R & I \\
I & \left(\frac{P}{\rho}\right)^{-1}
\end{array}\right] \geq 0 .
$$

Constraint (b) is equivalent to

$$
\begin{aligned}
\left(\frac{P}{\rho}\right)^{-1} & \left(A_{c l}+B_{c l}\left(V_{i} C_{c l}+V_{i}^{-} H\right)\right)^{T} \\
& +\left(A_{c l}+B_{c l}\left(V_{i} C_{c l}+V_{i}^{-} H\right)\right)\left(\frac{P}{\rho}\right)^{-1}<0
\end{aligned}
$$

for all $V_{i} \in \mathbf{V}$.

Condition (c) is equivalent to

$$
\rho h_{j} P^{-1} h_{j}^{T} \leq 1 \Leftrightarrow\left[\begin{array}{cc}
1 & h_{j}\left(\frac{P}{\rho}\right)^{-1} \\
\left(\frac{P}{\rho}\right)^{-1} h_{j}^{T} & \left(\frac{P}{\rho}\right)^{-1}
\end{array}\right] \geq 0 .
$$

for all $j \in\left[1, n_{u}\right]$, where $h_{j}$ be the $j$ th row of $H$.

If $X_{R}$ is a polyhedron, then from (9), (10), (11), the optimization problem (7) is equivalent to

$$
\begin{aligned}
& \inf _{Q>0, G} \gamma \\
& \text { s.t. } \quad(a 1)\left[\begin{array}{cc}
\gamma & x_{e}^{T} \\
x_{e} & Q
\end{array}\right] \geq 0, e \in[1, l] \\
& \quad(b 1) Q A_{c l}^{T}+A_{c l} Q+B_{c l} V_{i} C_{c l} Q+Q\left(B_{c l} V_{i} C_{c l}\right)^{T} \\
& \quad+B_{c l} V_{i}^{-} G+G^{T}\left(V_{i}^{-}\right)^{T} B_{c l}^{T}<0, i \in\left[1,2^{n_{u}}\right] \\
& \quad(c 1)\left[\begin{array}{cc}
1 & g_{j} \\
g_{j}^{T} & Q
\end{array}\right] \geq 0, j \in\left[1, n_{u}\right]
\end{aligned}
$$

where $\gamma=1 / \alpha^{2}, Q=\left(\frac{P}{\rho}\right)^{-1}$ and $G=H Q$. Let $g_{j}$ be the $j$ th row of $G$. It is easy to see that all constraints are given in LMIs. If $X_{R}$ is an ellipsoid, we need only to replace (a1) with

$$
\left[\begin{array}{rr}
\gamma R & I \\
I & Q
\end{array}\right] \geq 0
$$

\section{Controller Design}

In this section we will design a dynamic output feedback controller (4) such that the estimated domain of attraction is maximized with respect to $X_{R}$.

The following lemma given will be used in the sequel. Denote by $N_{\bar{B}}, N_{\bar{C}}$ any matrices whose columns form bases of the null space of $\bar{B}$ and $\bar{C}$, respectively.

$$
\begin{aligned}
\Gamma= & N_{\bar{c}}^{T} \Pi B_{c l}\left(I-V_{i}\right), \Gamma_{0}=N_{\bar{c}}^{T} \Pi_{0} B_{c l}\left(I-V_{i}\right), \\
W= & N_{\bar{B}}^{T} B_{c l}\left(I-V_{i}\right), \\
\Phi= & N_{\bar{c}}^{T}\left(\Pi A+A^{T} \Pi\right) N_{\bar{C}}-\Gamma \Gamma_{0}^{T}-\Gamma_{0} \Gamma^{T}+\Gamma_{0} \Gamma_{0}^{T} \\
& -N_{\bar{c}}^{T} H^{T} H_{0} N_{\bar{C}}-N_{\bar{c}}^{T} H_{0}^{T} H N_{\bar{C}}+N_{\bar{c}}^{T} H_{0}^{T} H_{0} N_{\bar{C}}, \\
\Theta= & N_{\bar{B}}^{T}\left(A Q+Q A^{T}\right) N_{\bar{B}}-W H H_{0}^{T} W^{T}-W H_{0} H^{T} W^{T} \\
& +W H_{0} H_{0}^{T} W^{T}-N_{\bar{B}}^{T} Q Q_{0} N_{\bar{B}}-N_{\bar{B}}^{T} Q_{0} Q N_{\bar{B}}+N_{\bar{B}}^{T} Q_{0} Q_{0} N_{\bar{B}} .
\end{aligned}
$$

where $\Pi=P / \rho$,

$$
A=\left[\begin{array}{cc}
A_{p} & 0 \\
0 & 0
\end{array}\right], \bar{B}^{T}=\left[\begin{array}{cc}
0 & B_{p} V_{i} \\
I & 0
\end{array}\right], \bar{C}=\left[\begin{array}{cc}
0 & I \\
C_{p} & 0
\end{array}\right]
$$

Lemma 1 For matrix variables $\Pi, Q, H$, the following statements hold:

(i) Constraint (b1) is equivalent to the constraints (b2) as follows

$$
\begin{gathered}
(b 2)\left[\begin{array}{cc}
\Phi & \Gamma+N_{\bar{c}}^{T} H^{T} \\
\left(\Gamma+N_{\bar{c}}^{T} H^{T}\right)^{T} & -I
\end{array}\right]_{i}<0, i \in\left[1,2^{n_{u}}\right] \\
{\left[\begin{array}{cc}
\Theta & W H+N_{\bar{B}}^{T} Q \\
\left(W H+N_{\bar{B}}^{T} Q\right)^{T} & -I
\end{array}\right]_{i}<0, i \in\left[1,2^{n_{u}}\right]} \\
\Pi Q=I
\end{gathered}
$$

(ii) Constraint (c1) is equivalent to constraint (c2) as follows

$$
\left[\begin{array}{cc}
\Xi & \Delta+\Lambda^{T} \\
\left(\Delta+\Lambda^{T}\right)^{T} & -I
\end{array}\right]_{j}<0, j \in\left[1, n_{u}\right]
$$

where $L_{j}$ be $1 \times n_{u}$ matrices whose element $l_{j}$ is 1 , and others are 0 , and

$$
\begin{aligned}
\Delta & =\left[\begin{array}{cc}
-L_{j} H & 0 \\
0 & 0
\end{array}\right], \quad \Lambda=\left[\begin{array}{ll}
0 & Q \\
0 & 0
\end{array}\right], \\
\Delta_{0} & =\left[\begin{array}{cc}
-L_{j} H_{0} & 0 \\
0 & 0
\end{array}\right], \Lambda_{0}=\left[\begin{array}{cc}
0 & Q_{0} \\
0 & 0
\end{array}\right], \\
\Xi & =\left[\begin{array}{cc}
-1 & 0 \\
0 & -Q
\end{array}\right]-\Delta \Delta_{0}^{T}-\Delta_{0} \Delta^{T}+\Delta_{0} \Delta_{0}^{T}-\Lambda^{T} \Lambda_{0} \\
& -\Lambda_{0}^{T} \Lambda+\Lambda_{0}^{T} \Lambda_{0} .
\end{aligned}
$$

\section{Proof}

Proof for (i): Obviously, the constraint (b1) can be rewritten as follows

$$
\begin{aligned}
& \Pi\left(A_{c l}+B_{c l} V_{i} C_{c l}+B_{c l}\left(I-V_{i}\right) H\right) \\
& +\left(A_{c l}+B_{c l} V_{i} C_{c l}+B_{c l}\left(I-V_{i}\right) H\right)^{T} \Pi<0 \\
\Leftrightarrow & \Pi\left(A+B_{c l}\left(I-V_{i}\right) H\right)+\left(A+B_{c l}\left(I-V_{i}\right) H\right)^{T} \Pi \\
& +\Pi \bar{B}^{T} K \bar{C}+\bar{C}^{T} K^{T} \bar{B} \Pi<0 .
\end{aligned}
$$


where

$$
K=\left[\begin{array}{ll}
A_{k} & B_{k} \\
C_{k} & D_{k}
\end{array}\right] .
$$

Then, (18) is equivalent to the following inequality.

$$
\begin{array}{cl}
(d 1) \quad N_{\bar{B}}^{T}\left(A+B_{c l}\left(I-V_{i}\right) H\right) \Pi^{-1} N_{\bar{B}} \\
\quad+N_{\bar{B}}^{T} \Pi^{-1}\left(A+B_{c l}\left(I-V_{i}\right) H\right)^{T} N_{\bar{B}}<0, \\
(d 2) \quad N_{\bar{C}}^{T} \Pi\left(A+B_{c l}\left(I-V_{i}\right) H\right) N_{\bar{C}} \\
\quad+N_{\bar{C}}^{T}\left(A+B_{c l}\left(I-V_{i}\right) H\right)^{T} \Pi N_{\bar{C}}<0,
\end{array}
$$

(d1) is equivalent to

$$
N_{\bar{B}}^{T}\left(A Q+Q A^{T}\right) N_{\bar{B}}+W H Q N_{\bar{B}}+N_{\bar{B}}^{T} Q H^{T} W^{T}<0 .
$$

Apparently, for any two matrices $X, Y$, the following equality always holds

$$
X Y+Y^{T} X^{T}=\left(X+Y^{T}\right)\left(X+Y^{T}\right)^{T}-X X^{T}-Y^{T} Y .
$$

In addition, as is known to all that for any matrix $V$, there always exists a matrix $V_{0}$ such that the following inequality holds

$$
\left(V-V_{0}\right)\left(V-V_{0}\right)^{T} \geq 0 .
$$

Thus, there exist $Q_{0}$ and $H_{0}$ such that (15) is equivalent to (20). On one hand, according to (22), if (20) holds, then (15) holds. On the other hand, when $Q_{0}=Q$ and $H_{0}=H$, (20) holds if (15) holds. And the similar conclusion can be drawn that $(\mathrm{d} 2) \Leftrightarrow(14)$. Thus, the proof for (i) is complete. Proof for (ii): (c1) is equivalent to

$$
\left[\begin{array}{cr}
-1 & 0 \\
0 & -Q
\end{array}\right]+\Delta \Lambda+\Lambda^{T} \Delta^{T} \leq 0 .
$$

The rest is similar to the proof of (i), and omitted here. Now, the whole proof is complete.

By solving inequalities $(a),(b 2),(c 2)$, we can solve Problem 2. But the constraints (b2) and (c2) are not LMIs, we can not solve them directly. To overcome this difficulty, we will give the following algorithm.

Algorithm 1 Let $\varepsilon>0$ be a given small constant specifying a convergence criterion.

Step 1 For system (2) design a standard dynamic output feedback controller $\Sigma_{k}$ such that the system (5) is asymptotic stable with saturation does not occur.

Step 2 Based on the given controller in Step 1 find a feasible set $\left(Q^{*}, H^{*}, \gamma^{*}\right)$ by solving the optimization problem (12). Let $\hat{\gamma}=\gamma^{*}+\eta$, where $\eta>\varepsilon$.

Step 3 Let $\bar{\gamma}=\eta, \hat{\gamma}=\gamma^{*}$. If $\bar{\gamma}<\varepsilon$, go to Step 11 .

Step 4 Let $k=0, t=0, \quad \tau=\tau_{0}$, where $\tau_{0}$ is a prescribed number, $\left(Q^{k}, H, \Pi^{k}, \gamma^{k}\right)=\left(Q^{*}, H^{*}, \Pi^{*}, \gamma^{*}\right)$, substitute the obtained matrix variables $\left(Q^{k}, H, \Pi^{k}, \gamma^{k}\right)$ into $\left(\Gamma_{0}, H_{0}, \Delta_{0}, \Lambda_{0}, \gamma\right)$.

Step 5 Solve the following LMI problem

$$
\begin{aligned}
\min & \operatorname{tr}\left(\Pi Q^{k}+Q \Pi^{k}\right)-2 n \\
& \text { subject to }(a),(b 2),(c 2) \\
& \text { and }\left[\begin{array}{cc}
\Pi & I \\
I & Q
\end{array}\right] \geq 0
\end{aligned}
$$

Step 6 If there do not exist matrices $\Pi, Q$ and $H$ satisfying (a), (b2) and (c2), let $\tau=\frac{1}{2} \tau, \gamma^{k}=\gamma^{*}, \gamma^{k}=\gamma^{k}-\frac{1}{2^{t}} \tau$, go to Step 5.

Step 7 Substitute the obtained matrix variables $(Q, H)$ into (a1), (b2) and (c). If condition (a1), (b2), (c) are satisfied, let $\left(Q^{*}, H^{*}, \Pi^{*}\right)=\left(Q, H, Q^{-1}\right),(\tilde{Q}, \tilde{\Pi})=\left(Q^{k}, \Pi^{k}\right), \eta=\gamma^{*}-\gamma^{k}$, $\gamma^{*}=\gamma^{k}, \gamma^{k}=\gamma^{k}-\frac{1}{2^{t}} \tau$, then go to Step 5 .

Step 8 If $k>N$ and $t>T$, where $N$ and $T$ are the maximum numbers of iterations allowed, go to Step 3 .

Step 9 If $k>N$, let $k=0,\left(Q^{k}, \Pi^{k}\right)=(\tilde{Q}, \tilde{\Pi}), t=t+1$, $\gamma^{k}=\gamma^{*}$, go to Step 5 .

Step 10 Let $k=k+1$ and $\left(Q^{k}, \Pi^{k}\right)=(Q, \Pi)$, go to Step 5 .

Step 11 Substitute the obtained matrix variables $(\Pi, H)$ into (18), obtain the controller gain matrix $K$, exit.

Remark 2 The above iteration algorithm is modified from Algorithm 1 in [28], which has been used in some previous works [27]. The convergence of the algorithm has been well investigated in [28]. By large number of examples, it is found that this algorithm generally performs well.

\section{EXAMPLES}

Example 1. Consider the system of the form (2) with

$$
A_{p}=\left[\begin{array}{ll}
0 & 1 \\
1 & 0
\end{array}\right], B_{p}=\left[\begin{array}{l}
0 \\
5
\end{array}\right], C_{p}=\left[\begin{array}{ll}
1 & 0
\end{array}\right],
$$

and a given standard dynamic output feedback controller with the gains described as

$$
A_{k}=-30, B_{k}=-22, C_{k}=-20, D_{k}=-30 .
$$

Let $R=I_{3 \times 3}$, by solving the optimization problem (12), we obtain $\gamma^{*}=118.0139$ and

$$
\begin{aligned}
\Pi^{*} & =\left[\begin{array}{ccc}
109.1588 & -0.4927 & 29.8610 \\
-0.4927 & 1.3420 & -2.6067 \\
29.8610 & -2.6067 & 20.4395
\end{array}\right], \\
H^{*} & =\left[\begin{array}{lll}
-7.7212 & -0.7368 & -0.4017
\end{array}\right] .
\end{aligned}
$$

Fig.1 shows the estimate of domain of attraction by quadratic Lyapunov function (ellipsoid) and four planes $\left(h_{1} x= \pm 1, C_{c l 1} x= \pm 1\right)$. Obviously, the ellipsoid $\alpha^{*} X_{R} \subset$ $\wp(H)$ and $\alpha^{*} X_{R} \not \subset \wp\left(C_{c l}\right)$.

Example 2. Consider the system described in Example 1, set $\varepsilon=2, N=5, T=5, \tau=5$, by Algorithm 1 with $\Pi^{*}, H^{*}, \gamma^{*}$ obtained in Example 1, we obtain $\gamma_{1}^{*}=56.9202$ and

$$
\begin{aligned}
K & =\left[\begin{array}{ccc}
-33.4815 & -133.8522 \\
-2.1163 & -11.8956
\end{array}\right], \\
H_{1}^{*} & =\left[\begin{array}{llc}
-7.0427 & -0.3701 & -0.3873
\end{array}\right], \\
\Pi_{1}^{*} & =\left[\begin{array}{ccc}
56.5258 & -0.0227 & 5.4276 \\
-0.0227 & 2.1547 & -0.5192 \\
5.4276 & -0.5192 & 1.5738
\end{array}\right] .
\end{aligned}
$$

Fig.2 shows the estimate of domain of attraction by quadratic Lyapunov function (ellipsoids). Obviously, $\alpha^{*} X_{R} \subset$ $\alpha_{1}^{*} X_{R}$. From Figure 2, we can see the efficiency of our design method. 


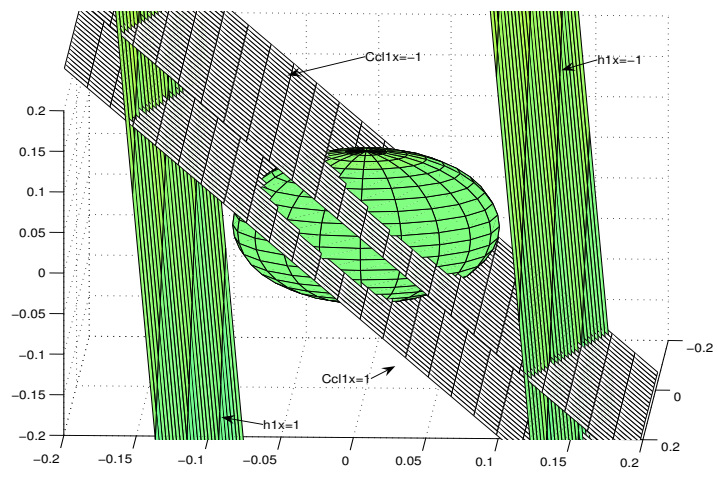

Fig. 1. Illustration of the condition for Example 1.

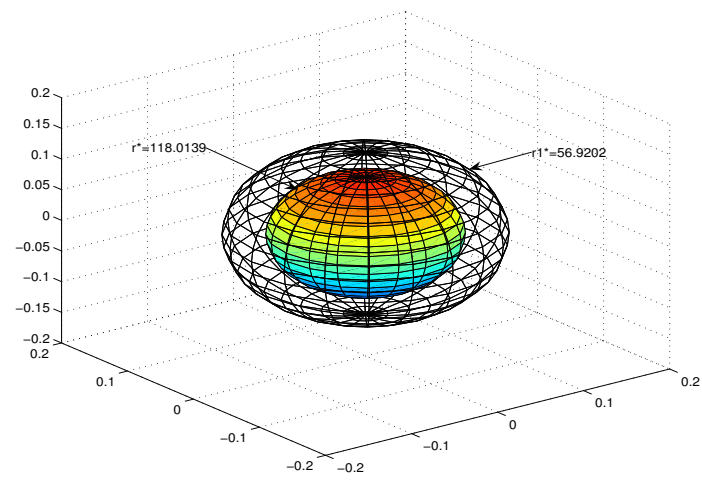

Fig. 2. Illustration of the condition for Example 1.

\section{CONCLUSIONS}

In this paper, a dynamic output feedback controller design approach based on cone complementary linearisation procedure has been proposed for linear time-invariant (LTI) systems with actuator saturation. Firstly, the estimation of domain of attraction has been given. Then based on this result, we have developed a controller design method to increase stability region. Examples have been used to demonstrate the effectiveness of these methods.

\section{REFERENCES}

[1] D. S. Bernstein, A. N Michel. A chronological bibliography on saturating actuators. International Journal of Robust and Nonlinear Control, 1995, 5(5): 375-380.

[2] Z. Lin. Low gain feedback. London: Springer, 1998.

[3] T. Hu, Z. Lin. Control systems with actuator saturation: Analysis and design. Boston: BirkhTauser, 2001.

[4] H. Chen, F. Allgöwer. A quasi-infinite horizon nonlinear model predictive control scheme with guaranteed stability. Automatica, 1998, 34(10): 1205-1218.

[5] D. Q. Mayne, J. B. Rawlings, C. V. Rao, and P. O. M. Scokaert. Constrained model predictive control: stability and optimality. Automatica, 2000, 36: 789-814.

[6] W. Chen, D. Ballance, J. OReilly. Optimisation of attraction domains of nonlinear MPC via LMI methods. Proceedings of the 2001 IEEE American Control Conferences. Arlington: IEEE, 2001: 3067-3072.

[7] J. De Don'a, A. Seron, M. M. Mayne, D. Q., G. C. Goodwin. Enlarged terminal sets guaranteeing stability of receding horizon control. Systems \& Control Letters, 2000, 47: 57-63.

[8] D. Limon, T. Alamo, E. F. Camacho. Stable constrained MPC without terminal constraint. Proceedings of the 2003 IEEE American Control Conferences. Denver: IEEE, 2003: 4893-4898.
[9] L. Magni, De Nicolao. G., L. Magnani, R. Scattolini. A stabilizing model-based predictive control algorithm for nonlinear systems. Automatica, 2001, 37: 1351-1362.

[10] M. Cannon, V. Deshmukh, B. Kouvaritakis. Nonlinear model predictive control with polytopic invariant set. Automatica, 2003, 39: 14871494.

[11] J.M. Gomes da Silva Jr, S. Tarbouriech. Anti-windup design with guaranteed regions of stability: an LMI-based approach. IEEE Transactions on Automatic Control, 2005, 50(1): 106-111.

[12] G. Grimm, J. Hatfi eld, I. Postlethwaite, A.R. Teel, M.C. Turner, and L. Zaccarian. Antiwindup for stable linear systems with input saturation: an LMI-based synthesis. IEEE Transactions on Automatic Control, 2003, (A), 48(9): 1509-1525.

[13] F. Wu, B. Lu. Anti-windup control design for exponentially unstable LTI systems with actuator saturation. Systems and Control Letters, 2004, 52(3-4): 304-322.

[14] D. Dai, T. Hu, A. R. Teel, L. Zaccarian. Control of saturated linear plants via output feedback containing an internal deadzone loop. Proceedings of the 2006 American Control Conferences. Minneapolis: IEEE, 2006: 5239-5245.

[15] J. M. Gomes da Silva, Jr., S. Tarbouriech, and R. Reginatto. Analysis of regions of stability for linear systems with saturating inputs through an anti-windup scheme. Proceedings of the 2002 IEEE Conf. Control Applications (CCA/CACSD02), Glasgow: IEEE, 2002: 1106-1111.

[16] G. Grimm, A.R. Teel, L. Zaccarian. Linear LMI-based external antiwindup augmentation for stable linear systems. Automatica, 2004, (B), 40(11): 1987-1996.

[17] L. Zaccarian, A. Teel. Nonlinear scheduled anti-windup design for linear systems. IEEE Transactions on Automatic Control, 2004, 49: 2055-2061.

[18] F. Blanchini. Set invariance in controlła survey. Automatica, 1999, 35(11): 1747-1767.

[19] P. dAlessandro, E. De Santis. Controlled invariance and feedback laws. IEEE Transactions on Automatic Control, 2001, 46: 1141-1146.

[20] E.G. Gilbert, K.T. Tan. Linear systems with state and control constraints: the theory and application of maximal output admissible sets. IEEE Transactions on Automatic Control, 1991, 36: 1008-1020.

[21] H. Hindi, S. Boyd. Analysis of linear systems with saturating using convex optimization. Proceedings of the 37th IEEE Conference on Decision and Control, Florida: IEEE, 1998: 903-908.

[22] Y. Cao, Z. Lin, D. G. Ward. An antiwindup approach to enlarging domain of attraction for linear systems subject to actuator saturation. IEEE Transactions on Automatic Control, 2002, 47: 140-145.

[23] T. Hu, Z. Lin, B.M. Chen. An analysis and design method for linear systems subject to actuator saturation and disturbance. Automatica, 2002, 38(2): 351-359.

[24] S. Boyd, L. El Ghaoui, E. Feron, V. Balakrishnan. Linear matrix inequalities in systems and control theory. SIAM Studies in Applied Mathematics series, Philadelphia: SIAM, 1994.

[25] E. J. Davison, E. M. Kurak. A computational method for determining quadratic Lyapunov functions for non-linear systems. Automatica, 1971, 7: 627-636.

[26] C. Pittet, S. Tarbouriech, C. Burgat. Stability regions for linear systems with saturating controls via circle and Popov criteria. Proceedings of the 36th IEEE Conference on Decision and Control, San Diego: IEEE, 1997: 4518-4523.

[27] Y.S. Moon, P. Park, W.H. Kwon, Y.S. Lee. Delay-dependent robust stabilization of uncertain state-delayed systems. International Journal of Control, 2001, 74: 1447-1455.

[28] L. El Ghaoui, F. Oustry, M. Ait Rami. A cone complementarity linearization algorithm for static output-feedback and related problems. IEEE Transactions on Automatic Control, 1997, 42(8): 1171-1176. 